\title{
EL MODELO DE CONTROL DE GESTIÓN MIGRATORIA FRANCÉS: UNA EXTENSIÓN DEL SISTEMA PENAL
}

\author{
Natalia Debandi ${ }^{1}$
}

\begin{abstract}
Este artículo se desprende de la tesis doctoral realizada sobre las políticas y prácticas de expulsión de migrantes en Francia. En primer lugar, se propone la expulsión de extranjeros como un dispositivo de seguridad ${ }^{2}$ que se concentrará especialmente en una parte de la población, los migrantes, catalogados como necesarios o excedente en función de los lineamientos que impone el mercado. Este dispositivo post-disciplinario se puede concebir como parte del sistema penal, conjugando con éste mecanismos y modalidades de intervención al mismo tiempo que se deslinda del peso burocrático y simbólico que éste ha adquirido. Esto se comprueba por ejemplo con la trasferencia del "publico" de un mecanismo al otro, es decir desde la prisión hacia los centros, a partir de la instauración del dispositivo de expulsión, pero también por medio de las prácticas y los innumerables puntos de contacto. Se señala asimismo el caso francés como un modelo de gestión migratoria que presenta características propias y distintivas de otras formas de gestión.
\end{abstract}

Palabras clave: detención, expulsión, criminalización de migrantes, retención, deportación, políticas migratorias.

Este artículo propone analizar los mecanismos de poder que se ponen en juego para el control social de la población extranjera en los países desarrollados, tomando como caso de estudio Francia. Analizar estos mecanismos, especialmente aquellos de carácter punitivo, permite comprender hacia donde se dirigen las actuales democracias neoliberales y qué clase de individuos y de lazos sociales se busca construir. Como diría Pilar Calveiro:

Es posible pensar que la observación de los sistemas punitivos actuales - la economía política de los cuerpos que instauran, sobre quienes recaen las penas, qué castigan y las tecnologías específicas que utilizan - nos permitirá

1 Instituto Gino Germani - UBA. Buenos Aires, Argentina.

2 FOUCAULT, Michel. Seguridad, territorio, población: Curso en el Collége de France, 1977-1978. 
iluminar al menos un fragmento de la anatomía política de nuestros tiempos: el que se ejerce en las actuales democracias de corte neoliberal ${ }^{3}$.

Este trabajo se desprende de la investigación realizada en la tesis doctoral cuyo objeto fue el retorno forzado en Francia entre el 2000 y el 2010 y para la cual se realizó un vasto trabajo de campo etnográfico. En primer lugar se trabajó durante un año como voluntaria en una permanencia de asistencia jurídica de extranjeros en instancia de expulsión (pero en libertad), posteriormente se trabajó como asistente jurídica dentro de un centro de retención en las cercanías de París. Asimismo se realizaron entrevistas a empleados y abogados de distintos centros de retención y se procedió durante varios meses a observación de audiencias para la expulsión de inmigrantes ${ }^{4}$.

Los centros de retención, las órdenes de expulsión y la sobrerrepresentación de extranjeros dentro del sistema penal forman parte del mismo mecanismo de control social, constituyéndose como la transformación de las prácticas y políticas penales en su afán por esculpir una fuerza de trabajo dócil y laboriosa: "A lo largo de las líneas de una relación históricamente contingente con el desarrollo de estructuras capitalistas de acumulación, ideologías, prácticas e instituciones de castigo contribuirían entonces a la reproducción de una fuerza de trabajo disciplinada" ${ }^{\prime 5}$.

Se puede pensar que la evolución de las políticas y prácticas en torno a los migrantes se funda sobre ese racismo de identidad que según Wieviorka "es de orden cultural y envía de forma particular a la manera en la cual la identidad nacional se retracta hoy en día sobre ella misma perdiendo su carda de universalidad, de apertura"6. La expulsión de migrantes constituye un engranaje más del Estado Penal ${ }^{7}$ que tomará los barrios o ghettos, las cárceles y finalmente los centros de expulsión como parte un complejo dispositivo de administración y control del excedente social ${ }^{8}$, que se amalgama con los árabes, negros, pobres, desocupados, mendigos y nómadas. El "problema" trasciende la extranjeridad, sin embargo la población extranjera será aquella más fácilmente tratable, ya que será la única a la que se le puede aplicar la pena más eficaz para su desviación, la deportación, que como explicaba Foucault en relación a los tipos de castigos definidos originalmente por Beccaria constituía el castigo ideal:

3 CALVEIRO, Pilar. Violencias de estado- La guerra antiterrorista y la guerra contra el crimen como medios de control global.

4 Se realizó observación dentro del Tribunal de Gran Instancia de París, donde se confirma la detención dentro de los centros de retención y en los Tribunales Administrativos, quienes ratifican o rectifican la orden de expulsión y la situación de irregularidad de los extranjeros.

5 DE GIORGI, Alessandro. Control de la inmigración, post- fordismo y menor elegibilidad: una crítica materialista de la criminalización de la inmigración en Europa.

6 BARATS, Christine. Racisme et exclusion: entretien avec Michel Wieviorka. Traducción propia.

WACQUANT, Loïc. Las cárceles de la miseria.

${ }^{8}$ DE GIORGI, Alessandro. El Gobierno De La Excedencia: Postfordismo y control de la multitud. 
En primer lugar el castigo expresado en la afirmación: 'tu has roto el pacto social, no perteneces más al cuerpo de la sociedad, tú mismo te has colocado fuera del espacio de la legalidad, nosotros te expulsaremos del espacio social donde funciona esa legalidad'. Es la idea que se encuentra frecuentemente en estos autores - Beccaria, Bentham, etc. - de que en realidad el castigo ideal sería simplemente expulsar a las personas, exiliarlas, destinarlas o deportarlas, es decir, el castigo ideal sería la deportación ${ }^{9}$.

Hoy en día la deportación parece retomar su importancia en un mundo que busca colocar a los excedentes al margen de la sociedad, "aux bords du monde"10.

\section{El surgimiento del modelo administrativo de gestión de la población extranjera en Francia}

El sistema penal, pensado como el conjunto de discursos, prácticas e instituciones ocupados del ejercicio de control social punitivo del Estado, incluye a la justicia y al derecho penal como mecanismo regulatorio de la desviación; a la policía, como la responsable de ejecutar la regulación del orden normado y al sistema penitenciario como instrumento de castigo frente a la violación de la norma.

En las sociedades democráticas occidentales el derecho penal ocupa un lugar diferente al derecho administrativo: se ocupa de sancionar a las personas que ocasionan daños a otros o a la sociedad en su conjunto. El derecho administrativo en cambio tendrá como objetivo regular el funcionamiento de las instituciones públicas y la relación con sus "administrados". En el derecho penal podemos distinguir dos tipos de faltas: los delitos cometidos contra otros individuos que serán definidos entonces como víctimas y aquellas faltas que carecen de victima concreta y que son realizados contra la sociedad en general. Este último tipo de sanción suele ser cuestionada en las sociedades contemporáneas, especialmente debido a la falta de consenso sobre el verdadero daño que ocasiona a una sociedad el delito cometido. El caso ejemplar es el del consumo de drogas, para el cual no existe un consenso sobre el perjuicio real que ocasiona a la sociedad en su conjunto y donde en todo caso el principal perjudicado sería el mismo individuo que comete la infracción ${ }^{11}$. Las faltas que cometen los extranjeros al encontrarse en situación irregular, ya sea ingresar al territorio de forma clandestina o mantenerse en este sin permiso de residencia, entrarían en esta tipología ya que no ocasionan perjuicio a ninguna persona concreta, sino que en todo caso afectaran - y tampoco será una posición consensuada - a la integridad de las fronteras nacionales. Hoy el tratamiento ante las infracciones relacionadas con la

\footnotetext{
9 FOUCAULT, Michel. La vérité et les formes juridiques.

${ }^{10}$ AGIER, Michel. Aux bords du monde, les réfugies. Paris: Flammarion,2002.

11 COMMISSAIRE AUX DROITS DE L'HOMME. La criminalisation des migrations en Europe: quelles incidences pour les droits de l'homme? Conseil de l'Europe, 2009.
} 
permanencia o ingreso irregular de extranjeros en Europa se reparte entre ambos derechos, el administrativo y el penal, variando las proporciones entre uno y otro según el Estado.

En Francia el encierro y expulsión de extranjeros era una medida penal que se sustentaba en la protección del Estado, es decir se concebía como medida de defensa de la seguridad nacional. En ese entonces, el extranjero indeseable era considerado sospechoso y en clave de defensa se lo hacía ingresar al sistema penal, desde el cual sería expulsado a su país de procedencia o mantenido al margen. Sin embargo esta concepción no era sistemática hacia toda la población migrante sino que sirvió históricamente para tratar afluencias puntuales, grupos específicos o casos individuales.

La relación simbiótica entre sistema penal y sistema administrativo, o entre derecho penal y derecho administrativo, se expresa en la diversidad de canales de intercambio y que a pesar de las enormes modificaciones que se han dado en los últimos veinte años remiten invariablemente a la dependencia estructural que entre ambas instituciones existe. Por un lado a partir de que se instaura a fines de los setenta la inmigración como una problemática política y discursiva en Francia, la población extranjera dentro del sistema penitenciario se mostrará en aumento hasta llegar a 1994 a la cifra record de 30\% de extranjeridad dentro de las prisiones francesas, es decir, hasta ese entonces el mecanismo privilegiado para el tratamiento de la población extranjera percibida como excedente era definitivamente el sistema penal en su concepción tradicional.

A partir de 1994 estos valores empiezan a descender hasta alcanzar un valor actual del 17\% (ver Tabla 1). El descenso es directamente proporcional a la creación y desarrollo de los Centros de Retención Administrativa (CRA) o centros de expulsión y la instauración de un sistema de expulsión administrativo presentado bajo un modelo "higiénico" y correcto, lo que permitirá fácilmente su aceptación social, se profundizará este aspecto más adelante. Cabe destacar que el año en el que se inicia la disminución de encarcelamiento de extranjeros dentro del sistema penal - 1994 - se crea una oficina específica para el control migratorio e inicia la intervención de la asociación civil dentro de los centros. La expulsión de extranjeros se transforma así en un verdadero mecanismo autónomo y permanente, que comenzará a recibir parte de la población que el sistema penitenciario hasta entonces absorbía. Esto ocurre en las vísperas de la puesta en marcha del tratado de Schengen (1995) que además de introducir la presión que la Unión Europea ejercía a cada país para el control interno de sus poblaciones migrantes y de sus fronteras exteriores, incorporaba en los países más ricos entre ellos Francia - el fantasma de una "invasión" desde los otros países, no solo de los ciudadanos originales de esas naciones sino de sus residentes temporales. 
Tabla 1 - Encierro de extranjeros en France 1990-2010

\begin{tabular}{|c|c|c|c|c|c|c|c|}
\hline & $\begin{array}{c}\text { (A) Pop. } \\
\text { total en } \\
\text { prisión }\end{array}$ & $\begin{array}{c}\text { (B) Pob. } \\
\text { extranjera } \\
\text { en prisión }\end{array}$ & $\begin{array}{c}\text { \% de Pob. } \\
\text { extranjera } \\
\text { en prisión }\end{array}$ & $\begin{array}{c}\text { (C) Cantidad } \\
\text { de } \\
\text { detenciones }\end{array}$ & $\begin{array}{c}\text { (D) Pop. } \\
\text { Extranjera } \\
\text { encerrada } \\
\text { (B +C) }\end{array}$ & $\begin{array}{c}\% \text { de } \\
\text { encierro de } \\
\text { extranjeros } \\
\text { (D/(A+C)) }\end{array}$ & $\begin{array}{c}\text { Tasa de } \\
\text { encierro } \\
\text { administrativo } \\
\text { de extranjeros } \\
\text { (C/D) }\end{array}$ \\
\hline 1990 & 43913 & 13026 & $29,70 \%$ & & & & \\
\hline 1991 & 47160 & 14343 & $30,40 \%$ & & & & \\
\hline 1992 & 50115 & 15044 & $30 \%$ & & & & \\
\hline 1993 & 50342 & 15507 & $30,80 \%$ & & & & \\
\hline 1994 & 52551 & 15921 & $30,30 \%$ & & & & \\
\hline 1995 & 53935 & 15362 & $28,50 \%$ & & & & \\
\hline 1996 & 55062 & 15674 & $28,50 \%$ & & & & \\
\hline 1997 & 54269 & 15120 & $27,90 \%$ & & & & \\
\hline 1998 & 53845 & 13557 & $25,20 \%$ & & 26851 & $39,94 \%$ & $53,11 \%$ \\
\hline 1999 & 52961 & 12591 & $23,50 \%$ & 14260 & $26 \% 10$ & $42,42 \%$ & $60,81 \%$ \\
\hline 2000 & 51441 & 11527 & $22,40 \%$ & 17883 & 29410 & \\
\hline 2001 & 47837 & 10234 & $21,40 \%$ & 16291 & 26525 & $41,36 \%$ & $61,42 \%$ \\
\hline 2002 & 48594 & 10507 & $21,60 \%$ & & & & \\
\hline 2003 & 55407 & 11918 & $21,50 \%$ & 28220 & 40138 & $48,00 \%$ & $70,31 \%$ \\
\hline 2004 & 59246 & 13123 & $22,20 \%$ & 25849 & 38972 & $45,80 \%$ & $66,33 \%$ \\
\hline 2005 & 59522 & 12467 & $20,90 \%$ & 30707 & 43174 & $47,85 \%$ & $71,12 \%$ \\
\hline 2006 & 60403 & 12284 & $20 \%$ & 30923 & 43207 & $47,31 \%$ & $71,57 \%$ \\
\hline 2007 & 64003 & 12160 & $19 \%$ & 34379 & 46539 & $47,30 \%$ & $73,87 \%$ \\
\hline 2008 & 66178 & 11927 & $18 \%$ & 32268 & 44195 & $44,89 \%$ & $73,01 \%$ \\
\hline 2009 & 66089 & 11652 & $17,60 \%$ & 35557 & 47209 & $46,44 \%$ & $75,32 \%$ \\
\hline 2010 & 66975 & 11767 & $17,50 \%$ & 33692 & 45459 & $45,16 \%$ & $74,12 \%$ \\
\hline
\end{tabular}

Fuente: elaboración propia a partir de Estudio de la CNCDH sobre extranjeros en prisión (Hasta el 2004), Anuario estadístico de la Justicia 2011 (2004-2010) e Informes anuales sobre los locales y centros de retención de la Cimade 2006-2010.

En la tabla 1 se puede observar que es a partir del año 2000 cuando el valor de extranjeridad dentro de las prisiones se estabiliza promediando el 19/20 por ciento. Lo que ocurre con la población extranjera será en primer lugar una transferencia desde el sistema penal hacia el sistema administrativo, aumentando considerablemente las detenciones administrativas al mismo tiempo que desciende la proporción de extranjeros en prisión. Sin embargo, no se trata exclusivamente de una transferencia sino del aumento neto de la cantidad de extranjeros privados de libertad. Si tomamos las poblaciones totales de ambos espacios de encierro, es decir, el total de personas detenidas en prisión por año más la cantidad de extranjeros encerrados en centros de expulsión y obtenemos el porcentaje de extranjeros privados de libertad, la tasa de encierro de extranjeros asciende a valores muy altos, $45 \%$ para el 2010 . Esto quiere decir que el $45 \%$ de las personas que son privadas de su libertad anualmente en Francia son extranjeros. Al mismo tiempo la tasa de encierro administrativo de extranjeros muestra el traspaso de buena parte de la población extranjera, pasando de un 53\% de extranjeros "tratados" por el sistema administrativo en 1999 al 74\% en el 2010. 
Por otro lado si observamos la cantidad de extranjeros encerrados en prisión entre 1990 y el 2010 se puede observar que dichas cifras no disminuyen drásticamente en su valor neto sino en su valor porcentual, esto se debe a que la población carceral francesa sufrirá al mismo tiempo de un aumento considerable, lo que muestra la tendencia punitiva general que se impone como metodología securitaria.

Gráfico 1 - Evolución de la población encarcelada en Francia 1999-2010

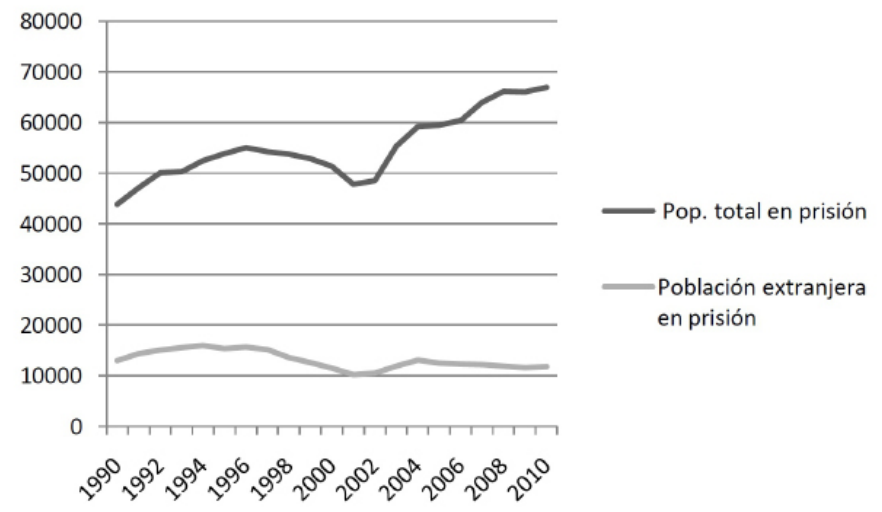

\section{Un modelo "aséptico" de control social}

La estrategia y el modelo punitivo francés en torno a los migrantes se apoyan en nuevos instrumentos, en herramientas asépticas de gestión del excedente y en este sentido constituye un nuevo formato de la economía del castigo. El dispositivo se instaura sobre un discurso despenalizador cuando en realidad esconde una estrategia represiva, propia del sistema penal. Este modelo podría pensarse como parte de esa transformación del Estado Social al Estado Penal propuesta por Wacquant ${ }^{12}$, aun cuando varios autores, entre ellos De Giorgi, insisten en la dificultad de amalgamar lo que ocurre en algunos países, como Estados Unidos o Brasil con el resto de las sociedades occidentales. En este sentido el "modelo francés" a diferencia de otros, será el de un Estado presente, es más, un estado que controlará de forma exhaustiva todo el campo social. Como ejemplo paradigmático de este modelo están las cites o guetos urbanos que lejos de vivir el "retiro"(repli) del Estado, vivirán una omnipresencia y muchas veces dependencia de las instituciones, las cuales sin embargo, no les ofrecerán a los habitantes del gueto salidas ni soluciones ${ }^{13}$; sino que más bien los condicionará a una distancia que los mantenga al margen del mundo social: "Los habitantes [del gueto] tienen el sentimiento que las instituciones trabajan en primer lugar para definir la distancia que ellos deben tener con la buena sociedad y a construir el

\footnotetext{
12 WACQUANT, op. cit.

${ }^{13}$ LAPEYRONNIE, Didier. Ghetto urbain, Ségrégation, violence, pauvreté en France aujourd'hui.
} 
problema"14. No se trata entonces tanto de la disminución o desaparición del Estado Social sino de la pérdida de legitimidad, de la incapacidad de integrar las poblaciones excluidas por el sistema neoliberal.

El control social estará definido en el modelo francés por el equilibro entre la mano izquierda y la mano derecha del Estado como decía Bourdieu, que distribuirán la población "excedente" entre los diversos mecanismos de relegamiento y control en función de la clasificación de peligrosidad que se ofrezca: en guetos o suburbios, en prisiones o deportados.

Los estudios foucaultianos proponen la cárcel como uno de los espacios donde mejor se pueden estudiar las características del poder que circula por el espacio social: "Esta perspectiva centra también su mirada en las funciones sociales de la cárcel en su contexto, en oposición a sus objetivos declarados, siendo las primeras las que podrían brindar una explicación sociológica del fenómeno, mientras que las últimas resultarán siempre en una mera justificación simbólica"15. Esta lectura que propone a la cárcel como un mecanismo de control social general de la población se puede extender al sistema de expulsión de extranjeros como una diversificación y especificación de este mecanismo, orientado a la población extranjera especialmente.

\section{De la ineficacia a la saturación del modelo}

La instauración de un modelo administrativo de expulsión pretende, en lo formal, la efectividad en materia de deportaciones, es decir, busca obtener el envío a sus países de origen de una mayor cantidad de extranjeros concebidos como excedente social. Este objetivo no es solo implícito sino que fue formalizado políticamente por el gobierno de Sarkozy que instauró "cifras objetivo" de deportaciones anuales sin que se conozca de antemano el porqué de estos valores ${ }^{16}$. Es decir, no solo se desconoce cómo fueron construidos sino el eventual impacto positivo que tendría un buen resultado. Estos valores son simbólicos y políticos, su valor cuantitativo consiste en una cifra que resulte emotiva numéricamente pero que al mismo tiempo pueda ser efectivamente obtenida por un poder político que busca instaurarse como el defensor de una cierta identidad francesa.

La cantidad neta de expulsiones ha venido en aumento desde la instauración efectiva del sistema administrativo de expulsión. Esta cantidad aumenta de forma abrupta a partir del 2005 cuando se instauran estos objetivos políticos y donde desde el Estado se da un fuerte impulso discursivo, político y económico al sistema de expulsión. La cantidad de retornos forzados desde Francia metropolitana pasa así de 7.186 en 1990 a 19.600 en el 2010.

\footnotetext{
14 Ibidem, traducción propia.

${ }^{15}$ DAROQUI, Alcira, LOPEZ, Ana Laura. La cadena punitiva:actores, discursos y prácticas enlazadas, p. 101-107.

${ }^{16}$ BORREL, Catherine. Des statistiques fragiles et incomplètes. Plein Droit, n. 77, juin 2008.
} 
No se debe sin embargo olvidar la dimensión humana del problema, más allá de los valores y de la eficiencia o no del método, los números se refieren a que 19 mil personas fueron extraídas por la fuerza de su lugar de residencia habitual dejando atrás sus trabajos, sus lazos familiares o sociales establecidos y cortando sus expectativas y ambiciones, no se trata salvo excepciones de devoluciones en frontera las cuales se contabilizan de otra manera. Por otro lado, estos valores que parecieran mostrar un éxito rotundo en materia de control migratorio, al enfrentarlos a un análisis exhaustivo acerca de los costos y la tasa de efectividad en función de los mecanismos involucrados, brindan un panorama menos optimista.

$\mathrm{Si}$ se estudian las proporciones de expulsiones efectivamente logradas en función del despliegue de instrumentos desarrollado, los valores son menos significativos (ver tabla 2). Por ejemplo, analizando la obligación dejar el territorio (OQTF), que consiste en las órdenes de expulsión enviadas por carta y que acompañan toda denegación de regularización, la cantidad alcanza cifras exorbitantes, creando un "batallón" de extranjeros en situación irregular y con valores de efectividad extremadamente bajos, aun si tomamos su mejor resultado desde su instauración (12\% en el 2009). Es decir, un valor de ejecución irrisorio en función de un despliegue extraordinario: más de 40 mil durante ese mismo año. Esta masificación de la expulsión por carta tendrá también un impacto económico, especialmente en el aumento exponencial de casos dentro del fuero administrativo así como el gasto en defensores públicos (aide juridictionnelle).

Tabla 2 - Medidas pronunciadas y tasa de ejecución 2006-2009

\begin{tabular}{|c|c|c|c|c|c|}
\hline \multirow{4}{*}{ APRF (1) } & Pronunciadas & 2006 & 2007 & 2008 & 2009 \\
\hline & Ejecutadas & 16616 & 11891 & 9844 & 10422 \\
\cline { 2 - 6 } & Tasa de ejecución & $25,70 \%$ & 23,4 & $22,40 \%$ & $26,00 \%$ \\
\hline \multirow{4}{*}{ OQTF (2) } & Pronunciadas & & 46263 & 42130 & 40191 \\
\cline { 2 - 6 } & Ejecutadas & & 1816 & 3050 & 4914 \\
\cline { 2 - 6 } & Tasa de ejecución & & $3,90 \%$ & $7,20 \%$ & $12,20 \%$ \\
\hline \multirow{3}{*}{ ITF (3) } & Pronunciadas & 4697 & 3580 & 2611 & 2009 \\
\cline { 2 - 6 } & Ejecutadas & 1892 & 1544 & 1386 & 1330 \\
\cline { 2 - 6 } & Tasa de ejecución & $40,30 \%$ & $43,10 \%$ & $53,10 \%$ & $66,20 \%$ \\
\hline \multirow{2}{*}{ Todas las medidas } & Pronunciadas & 11348 & 11138 & 12663 & 12162 \\
\cline { 2 - 6 } & Easa de ejecución & $27,70 \%$ & $17,90 \%$ & $19,40 \%$ & $30,90 \%$ \\
\hline
\end{tabular}


Fuentes: Elaboración propia a partir del Séptimo Informe sobre las orientaciones de la política de inmigración, marzo 2011 y el Informes de la Corte de cuentas del Senado (2009).

(1) Arreté prefectorale de reconduite a la frontiere: medida de expulsión dictada por lo general frente a un control migratorio de un extranjero en situación irregular. Estas medidas se asignan en general a partir de controles desplegados en las calles o barrios populares.

(2) Obligation a quitter le territoire français: corresponde a la orden de expulsión dictada como acompañamiento casi automático de todo rechazo de regularización. Tienen duración de un año y en ese lapso si no se recurre y la persona es detenida en un control migratorio permite la expulsión de forma automática.

(3) Interdiction du territoire français: es una medida dictada por un juez penal como pena sumplementaria a una condena de carácter penal. Impone una expulsión y el impedimento al reingreso por el período que se determine.

La interdicción al territorio (ITF) es la medida cuya tasa de ejecución resulta mejor, corresponde en su mayoría a personas deportadas desde la prisión, en cuyo caso la administración ha tenido tiempo de tramitar los salvoconductos o pasaportes y contarán con todos los trámites al momento de finalizar la condena. Sin embargo, numéricamente los ITF no resultan cuantitativamente significantes en relación a las otras medidas, representando apenas el 4,6\% ${ }^{17}$.

Para obtener la expulsión efectiva de los extranjeros el mecanismo privilegiado será el encierro en un centro de expulsión, sin embargo la eficacia de este método presenta una disminución porcentual en los últimos años, ya que si en el 2002 correspondía al 92\% de los retornos forzados para el 2010 este valor parece reducirse al 51\% (tabla 3). Los retornos forzados que no son realizados desde un centro de retención corresponden a modalidades de expulsión no muy claras y que no son explicitadas en los informes oficiales. Se puede suponer que serán individuos que son expulsados de forma inmediata al ser detenidos, es decir directamente desde las comisarías o desde los locales de retención administrativa o bien readmisiones entre los países vecinos. Este último caso parece estar en aumento, y se refiere particularmente a la detención de extranjeros en las fronteras interiores, es decir, la detención de individuos en las zonas aledañas a las fronteras francesas que se encuentren o bien ingresando o incluso saliendo del territorio. Estas readmisiones Schengen permiten enviar de forma inmediata a los extranjeros al país dentro de la Unión Europea por el que ingresó ${ }^{18}$ y aumentar fácilmente las cifras de expulsión. Lo que es fundamental recalcar es que a pesar de la disminución efectiva de extranjeros que son expulsados desde los centros de retención, la cantidad de individuos bajo encierro en estos espacios continúa en aumento, dando la pauta de que este modelo no tiene solo por objetivo la expulsión efectiva sino la instauración de un mecanismo de difusión del temor, "el miedo a ser expulsado", creando una población migrante dócil y auto-invisibilizada, que carezca de pretensiones y se contente con permanecer mientras pueda.

17 CIMADE. Centres et locaux de rétention administrative. Rapport 2010. Paris: La Cimade, 2010.

18 Ibidem. 
Tabla 3 - Tasa de expulsiones efectivas por número de detenciones

\begin{tabular}{|c|c|c|c|c|c|}
\hline & $\begin{array}{c}\text { Retornos } \\
\text { forzados }\end{array}$ & $\begin{array}{c}\text { Expulsados } \\
\text { desde CRA }\end{array}$ & $\begin{array}{c}\text { \% de expulsiones } \\
\text { desde CRA }\end{array}$ & $\begin{array}{c}\text { Cantidad de } \\
\text { detenciones por } \\
\text { control }\end{array}$ & $\begin{array}{c}\text { Tasa de expulsión } \\
\text { sobre detenciones }\end{array}$ \\
\hline 2002 & 10067 & 9384 & $93,22 \%$ & 49362 & $20,39 \%$ \\
\hline 2003 & 12482 & & & 59023 & $21,15 \%$ \\
\hline 2004 & 15660 & & & 64218 & \\
\hline 2005 & 19841 & & & 82824 & $24,80 \%$ \\
\hline 2006 & 22412 & 16909 & $75,45 \%$ & 90362 & $19,20 \%$ \\
\hline 2007 & 19885 & 15170 & $76,29 \%$ & 103556 & $17,66 \%$ \\
\hline 2008 & 19724 & 14411 & $73,06 \%$ & 111692 & $21,87 \%$ \\
\hline 2009 & 21020 & 11217 & $53,36 \%$ & 96109 & \\
\hline 2010 & 19600 & 10004 & $51,04 \%$ & & \\
\hline
\end{tabular}

Fuente : elaboración propia a partir de los Informes anuales sobre los locales y centros de retención elaborados por la Cimade (2005-2010), el Sitio web del Senado Francés y documentos de la Corte de cuentas del Senado (2009-2010).

El éxito relativo del modelo francés de expulsión se ve aun de forma más marcada si se tiene en cuenta la cantidad de detenciones por control ${ }^{19}$ necesarias para poder cubrir los objetivos de expulsión fijados. Se puede observar, si comparamos la cantidad de detenciones realizadas en el año 2003 - año previo a la instauración de una cuantificación política del objetivo a expulsar - con las del 2008, que el valor prácticamente se duplicó (tabla 3) pasando de 59.023 en el 2003 a 111.692 para el 2008 y experimentando un leve descenso en el 2009. Este valor en sí mismo representa una cifra abismal de detenciones, la tasa de efectividad sin embargo, se mantiene similar e incluso desciende en el 2008 a solo el 17,6\%. De esta manera, para el año 2009 las 96.109 detenciones Ilevaron a la expulsión efectiva de 21 mil personas aproximadamente, lo que significa que se deben detener más de 4 personas para lograr la repatriación de una. Al mismo tiempo si tenemos en cuenta que las personas que reciben la expulsión por carta y los que obtienen un ITF no forman parte necesariamente del grupo de los controlados y detenidos, podemos inferir a grosso modo, que el dispositivo de expulsión "toca" a más de 125 mil extranjeros anualmente ${ }^{20}$.

La detención masiva es una de las características y fundamentos del modelo francés y resulta al mismo tiempo el lado más oscuro de la metodología higiénica y correcta que se despliega. En primer lugar porque la forma en que

\footnotetext{
${ }^{19}$ En francés se refieren a interpelaciones, esto implica el control migratorio y la detención en comisaría.

${ }^{20}$ Es decir, calculando que cerca de 94 mil extranjeros son interpelados y que, por ejemplo para el 2009, 40 mil extranjeros recibieron un OQTF y de estos seguramente menos de 10 mil forman parte de los que fueron además interpelados (como referencia anualmente la cantidad de personas con OQTF en los centros no pasa de 2500), se puede pensar que más de 30 mil extranjeros no fueron interpelados pero fueron "victimas" del dispositivo de expulsión.
} 
se realizará este control es sin lugar a dudas sesgado - se realiza dentro de los barrios populares y a aquellos individuos cuya apariencia física los transforma en "sospechosos" - y porque existe una fuerza policial autorizada a la realización continua y periódica de controles cuyo único objetivo es captar extranjeros en situación irregular para nutrir el sistema de expulsión. En Francia la policía de fronteras (PAF) puede ir en busca de sus "clientes" y no solo en los espacios públicos sino en sus domicilios, lugares de trabajo o espacios administrativos, basados en el único motivo de sospecha de irregularidad. Este mecanismo absolutamente represivo será único en su forma en Europa y tampoco se presenta en otros lugares con fuerte control migratorio como los Estados Unidos, donde si bien pueden ocurrir eventualmente situaciones de detención de esta índole, es decir en los trabajos o domicilios, serán casos esporádicos o específicos relativos a "zonas problemáticas". La Tabla 4 muestra los tipos de detención en el 2010 de los extranjeros encerrados en los centros de retención donde se observa que más del $10 \%$ de la población detenida fue interpelada o bien en su domicilio, trabajo o dentro de alguna institución como el tribunal, la prefectura o la municipalidad.

Tabla 4 - Lugares de detención de extranjeros - 2010

\begin{tabular}{|c|c|c|}
\hline Controle en vía pública, estaciones o transportes públicos & 9.261 & $47 \%$ \\
\hline Controles generales de policía & 3.798 & $19,10 \%$ \\
\hline Control en frontera & 2.748 & $13,80 \%$ \\
\hline Prisión & 1.070 & $5,40 \%$ \\
\hline Domicilio & 1.244 & $6,30 \%$ \\
\hline Trabajo & 1.090 & $5,50 \%$ \\
\hline Al momento del casamiento civil & 29 & $0,10 \%$ \\
\hline En comisaría, prefectura o tribunal & 535 & $3 \%$ \\
\hline Denuncias & 116 & $0,60 \%$ \\
\hline TOTAL & 19.891 & $100 \%$ \\
\hline
\end{tabular}

Fuente: Reporte anual sobre los locales y centros de retención. Cimade 2010.

Para poder llevar a cabo un modelo capaz de detener esta cantidad de personas, se requiere de una organización sofisticada y de una inversión en capital económico y humano muy grande, no todos los países estarán en condiciones de realizar esto. En otros países se apoyarán directamente en el sistema penal. En Estados Unidos por ejemplo para el 2011 se deportaron 396.906 personas, pero el $90 \%$ de estas corresponden a quienes han trasgredido leyes criminales, acaban de cruzar la frontera ilegalmente, han trasgredido repetidamente las leyes 
de inmigración o son fugitivos de la corte de inmigración. Es decir, los criminales, los recién llegados y los reincidentes. Algunos de estos aspectos, especialmente la acentuación de la pena ante la reincidencia ha sido incorporado en las últimas modificaciones legislativas en Francia a raíz de la Directiva retorno impuesta en Europa desde el 2009. Otros, casos como España e Italia han buscado en los últimos años aproximarse en cierta manera al modelo francés, sin embargo el mayor esfuerzo consistirá en bloquear las fronteras externas, por lo que la población extranjera interna quedará menos custodiada y perseguida.

Retomando los valores anteriormente expuestos acerca de la cantidad de interpelaciones, detenciones en centros y expulsiones, lo que se puede observar es que el sistema alcanza su saturación en el 2006, donde se muestra que a pesar del aumento constante de las retenciones administrativas, la tasa de expulsión desde los centros es cada vez menor (Gráfico 2). Si bien en términos absolutos la cantidad de expulsiones continúa en aumento, es a partir de otros mecanismos, como las ayudas al retorno, que se logra incrementar y alcanzar los valores esperados. Según se puede observar en el Gráfico 3, los retornos forzados, realizados desde los centros o por otros mecanismos llegan a un punto claro de saturación en el 2006, momento en el cual se mantendrán constantes los valores.

Gráfico 2 - Evolución de las retenciones y de las expulsiones desde CRA 2003-2010

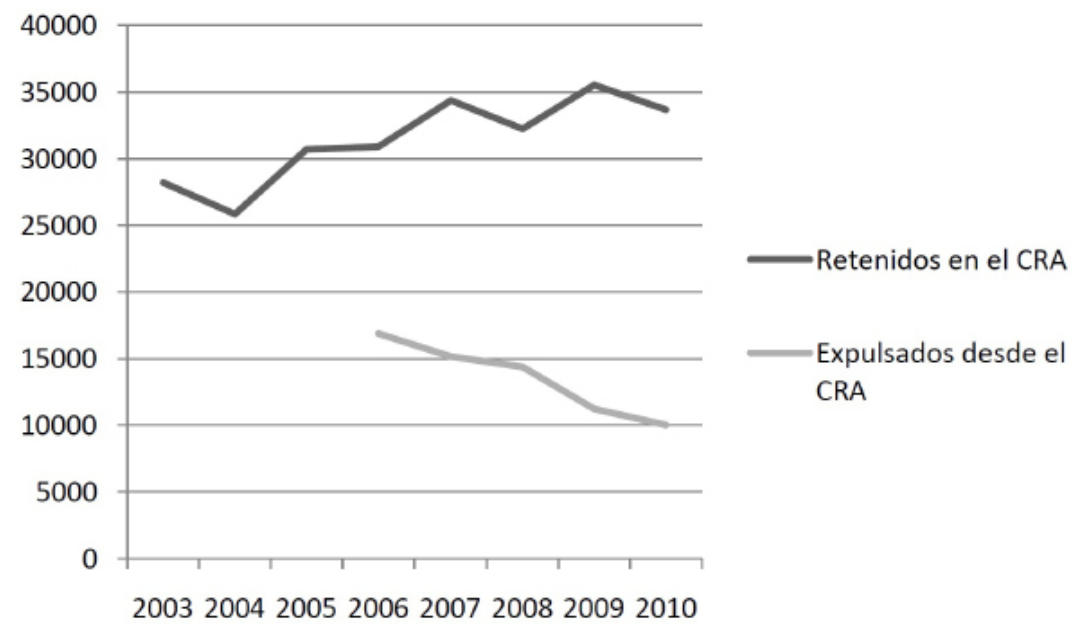

Fuente: CIMADE. 
Gráfico 3 - Evolución de las detenciones y de las expulsiones 2002-2010

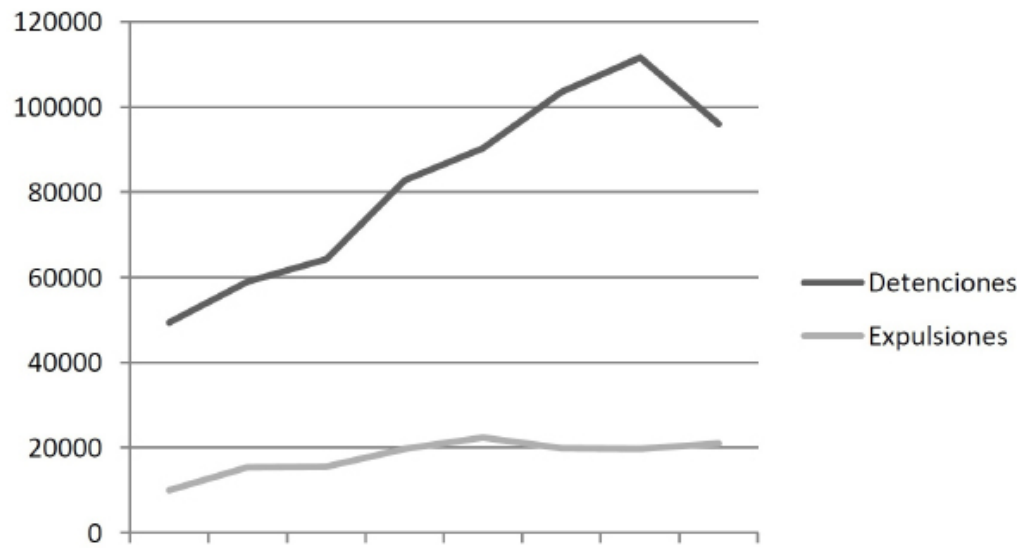

20022003200420052006200720082009

Fuente: Informes anuales sobre los locales y centros de retención de la Cimade 2006-2010.

A pesar de los años de experiencia, el modelo aún se encuentra en proceso de adaptación y de revisión, las idas y vueltas entre lo penal y lo administrativo no son más que una clara expresión de la búsqueda del equilibrio entre eficiencia, bajo costos y aceptación social. Las modificaciones legislativas incorporadas en el 2011, demostraron este retorno hacia lo penal, incorporando nuevamente y endureciendo aún más el tratamiento hacia la población migrante. Por ejemplo, el artículo 621-1 del código CESEDA establece que: "El extranjero que ha ingresado o permanecido en Francia sin acatar las disposiciones de los artículos L.211 y L311-1 o que se mantuvo en Francia más allá de la duración autorizada por su visa será penado de prisión de un año de duración y de una multa de 3750 euros $(\ldots)^{\prime \prime 21}$.

Esta medida habilita a tratar al extranjero irregular por cualquiera de los dos mecanismos, la retención administrativa y el sistema penal, equiparando automáticamente ambos sistemas. Otra innovación que va en este sentido, es el artículo 624-1 del Ceseda el cual determina que el extranjero que fue expulsado y que ingresa nuevamente en el territorio francés podrá ser sancionado por medio de una pena de hasta tres años de prisión, la misma pena que podrán recibir aquellos que eviten la deportación o que oculten sus documentos o identidades.

Estos artículos vienen claramente a solventar algunos de los principales huecos que el sistema de expulsión administrativo presentaba hasta entonces, por un lado el retorno de los migrantes expulsados, quienes, como ya hemos

${ }^{21}$ Artículo 621-1 delcódigo CESEDA (Code de l'Entrée et du Séjour des Etrangers et du Droit D'asile). El Cesada es el instrumento jurídico que regula la entrada y permanencia de los extranjeros en Francia así como el derecho a asilo. 
mencionado continuarán en su mayoría la aventura migratoria pesar de la expulsión y en segundo lugar, y principal, pretende transferir al sistema penal los inexpulsables. La gran mayoría de individuos no contará con sus documentos de viaje o no los presentará, y en función de la nueva legislación este solo argumento bastará para transferirlo al sistema penal, esto se desarrollaría de forma discrecional decidiendo en cada caso cual instrumento represivo conviene más en función de la nacionalidad, la trayectoria y los antecedentes, penales o administrativos del individuo.

Incluso las discusiones en teoría de carácter protector que sucedieron a estas medidas y que dieron lugar al pronunciamiento de la Corte de Justicia de la Unión Europea en contra de estas disposiciones no se basaron en los derechos de las personas y en las características no penales de la infracción migratoria sino en la eficacia del mecanismo administrativo, que por lo tanto deberá ser priorizado.

Finalmente la Corte de casación definió que el extranjero no podrá ser detenido en prisión exclusivamente por una medida de expulsión, salvo para el caso en que se trate de una reincidencia, es decir, cuando haya reingresado en el país luego de una expulsión o haya pasado por un centro de retención y la medida no haya podido ser ejecutada. Esto viene a incrementar de una manera drástica el castigo que recibe el extranjero que pasa por un centro de retención, el cual por el solo hecho de haber sido detenido y que la administración o su consulado no hayan sido capaces de lograr dicha deportación estará expuesto a una pena de prisión.

\section{Conclusiones}

Las prácticas y políticas de expulsión de migrantes pueden ser concebidas como un dispositivo, en los términos propuestos por Foucault, formando parte de un sistema de control social más amplio, tradicionalmente instaurado a partir del sistema penal. La cárcel ha sido históricamente el instrumento predilecto para la gestión de la población desviada y de forma extendida para "el desecho social"22 o "el excedente"23, la cual corresponde a los sectores sociales más pobres. En Francia - y en Europa en general - este sector, es decir, el pobre, está conformado en buena parte por población de origen extranjero, ya sea migrante o nacional que habilitará al estado a desplegar estrategias innovadoras y eficientes, entre ella la expulsión.

El modelo francés de expulsión de migrantes se presenta como un complemento "aséptico" y ordenado del sistema penal, fundando con éste un mismo mecanismo de represión y control social, en este caso, específico para la población extranjera. Francia no es el país con mayor tasa de expulsiones, por

\footnotetext{
${ }^{22}$ WACQUANT, op. cit.

${ }^{23}$ DE GIORGI, El Gobierno..., op. cit.
} 
lejos Estados Unidos e inclusive otros países de Europa realizan mayor número de retornos forzados, sin embargo la particularidad del modelo francés es la población a la cual está destinada y la complejidad del dispositivo instaurado. Estados Unidos, por ejemplo presenta cifras abismales de expulsiones, sin embargo la mayor parte de los individuos deportados proviene de la prisión. Otros países, como España o Italia presentan altos números de retornos forzados en frontera, las llamadas devoluciones (refoulement) y que en Francia no son tenidas en cuenta como expulsiones y se encuentran en disminución en los últimos años. Los extranjeros expulsados o en vía de expulsión en Francia son individuos que fueron capturados por fuerzas de seguridad que activamente fueron a buscar estos individuos - y no otros - en los espacios públicos y privados. Esta logística e inteligencia desplegada para la captura de extranjeros es un signo distintivo del modelo francés y que se diferencia de otros países de fuerte afluencia migrante.

\section{Bibliografía}

BARATS, Christine. Racisme et exclusion: entretien avec Michel Wieviorka. Quaderni, n. 22, 1994, p. 125-140.

BOURDIEU, Pierre. Las estrategias de la reproducción social. Buenos Aires: Editorial Siglo XXI Argentina, 2011.

CALVEIRO, Pilar. Violencias de estado. La guerra antiterrorista y la guerra contra el crimen como medios de control global. Buenos Aires: Siglo veintiuno editores, 2012.

DAROQUI, Alcira; LOPEZ, Ana Laura. La cadena punitiva: actores, discursos y prácticas enlazadas. In DAROQUI, Alcira; LOPEZ, Ana Laura; CIPRIANO GARCIA. Sujeto de Castigos. Buenos Aires: HomoSapiens, 2012, p. 101-107.

DE GIORGI, Alessandro. El Gobierno de la Excedencia: Postfordismo y control de la multitud. 1aㅡ ed. Madrid: Traficantes de Sueños, 2006.

DE GIORGI, Alessandro. Control de la inmigración, post-fordismo y menor elegibilidad: una crítica materialista de la criminalización de la inmigración en Europa. Critica penal y poder, n. 2, 2012, p. 139-162.

FOUCAULT, Michel. Seguridad, territorio, población: Curso en el Collége de France, 1977-1978. Buenos Aires: Fondo de Cultura Económica, 1973-1978/2006.

FOUCAULT, Michel. La vérité et les formes juridiques. Conférence à I'Université pontificale de Rio de Janeiro, 1973.

LAPEYRONNIE, Didier. Ghetto urbain, Ségrégation, violence, pauvreté en France aujourd'hui. Paris: Edition Robert Laffont, 2008.

MERA, Carolina; HALPERN, Gerardo. Migraciones internacionales: repensando las ciudades y sus políticas. Revista Latina de Sociología, 2011, p. 98-125.

SAYAD, Abdelmalek. L'immigration, ou les paradoxes de l'alterité. Paris: Raisons d'agir Editions, 2006.

WACQUANT, Loïc. Las cárceles de la miseria. Buenos Aires: Manantial, 1999. 


\section{Abstract \\ The French model for migratory control management: an extension of the penal system}

This article follows from the doctoral thesis on the policies and practices of expulsion of migrants in France. First, the expulsion of foreigners is proposed as a security dispositif ${ }^{24}$ that is especially concentrated in one part of the population: migrants, classified as necessary or surplus according to the guidelines imposed by the market. This post-disciplinary mechanism can be seen as part of the penal system, combining with it mechanisms and modalities of intervention while disclaims bureaucratic and symbolic weight that it has acquired. This is seen for example with the transfer of the "public" of a mechanism to another, from prison to the centers, since the establishment of the deportation system but also through practices and countless points of contact. The French case is also noted as a model of migration management or emblem that presents distinctive properties or characteristics from other forms of management features.

Keywords: detention, expulsion, criminalization of migrants, retention, deportation, migratory policies.

Recibido para publicación en 25/06/2015

Aceptado para publicación en 22/10/2015

Received for publication in June, $25^{\text {th }}, 2015$

Accepted for publication in October, 22 $2^{\text {th }}, 2015$

ISSN impresso 1980-8585

ISSN eletrônico 2237-9843

http://dx.doi.org/10.1590/1980-85852503880004506

$\overline{{ }^{24} \text { FOUCAULT, op. cit. }}$ 